\title{
Dynamics of Physical Autonomous Robotic Network With Constant And Time-Varying Communication Delays
}

Kuei-Fang Hsueh

University of Toronto

Isam Al-Darabsah

University of Waterloo

Mohammad Al Janaideh ( $\nabla$ maljanaideh@mun.ca )

University of Toronto

Sue Ann Campbell

University of Waterloo

Deepa Kundur

University of Toronto

Research Article

Keywords: Autonomous, flow, CAVN

Posted Date: December 22nd, 2021

DOI: https://doi.org/10.21203/rs.3.rs-1157959/v1

License: (a) (i) This work is licensed under a Creative Commons Attribution 4.0 International License.

Read Full License 


\title{
Dynamics of Physical Autonomous Robotic Network with Constant and Time-Varying Communication Delays
}

\author{
Kuei-Fang Hsueh *,† Isam Al-Darabsah $\$, \S \quad$ Mohammad Al Janaideh *, \\ Sue Ann Campbell $\ddagger$ N Deepa Kundur *,**
}

\begin{abstract}
A Connected Autonomous Vehicle Network (CAVN) is an emerging paradigm that can reduce traffic congestion by allowing vehicles to cooperatively behave according to information out of the line of sight and improve traffic flow by decreasing inter-vehicular gaps on roadways. In this paper, the stability of CAVN with constant and time-varying communication delays is studied. When the time delay depends on time, the semi-discretization method is used to study the plant stability of the flow equilibrium of CAVN and construct approximate stability regions charts over control gain space. To study the influence of time-varying delay, a constant delay is considered as the average value of the time delay function. Then, we provide explicit sufficient conditions for the stability of the flow equilibrium and study the string stability of the CAVN. The proposed controller is validated using simulations and experimentally with a platoon of four autonomous robots under time-varying delays.
\end{abstract}

\section{Introduction}

A Connected Autonomous Vehicle Network (CAVN) is a multiagent system that communicates and controls the speed and position of autonomous vehicles through a wireless exchange of information among the in-network vehicles. This new paradigm represents an emerging cyber-physical system (consisting of networking, computation, and physical processes) with significant potential to enhance vehicle safety, ease traffic congestion and positively impact the environment through autonomous platoon control $[1,2,3]$. The cyber component of such a system incorporates a vehicle-to-vehicle communication network, while the physical component of such a system includes vehicle dynamics and human-driver responses. Within CAVN, communication networks enable opportunities for greater situational awareness, collaborative decision-making, and improved control $[4,5]$.

*Edward S. Rogers Sr. Department of Electrical and Computer Engineering, University of Toronto, Toronto, ON M5S 3G4, Canada

${ }_{\dagger}^{\dagger}$ Email: albert.hsueh@ mail.utoronto.ca

${ }^{\ddagger}$ Department of Applied Mathematics at University of Waterloo, Waterloo, ON, N2L 3G1, Canada

$\S$ Email: ialdarabsah@uwaterloo.ca

IEmail: maljanaideh@mun.ca (Corresponding author)

"Email: sacampbell@uwaterloo.ca

${ }^{* *}$ Email: dkundur@ece.utoronto.ca 
CAVNs have attracted considerable attention during the last decade because of many technical challenges. These include developing effective strategies for automation, control, and communication as well as accounting for human factors in system design [6]. From the cyber perspective, one significant challenge is the presence of inter-vehicular communication delay that limits the accessibility to timely and accurate information necessary for real-time control and accurate decision-making[7]. In general, communication delays can be classified into two categories: naturally occurring or adversarial induced. Naturally occurring communication delays are inherent to the cyber or physical components of the system. They could be random delays (latency) in the vehicle to vehicle (V2V) infrastructure, represent processing delays in the technology stack incurred from synchronizing data from multiple sensors, or physical latency in the CAN bus. Recently, naturally occurring communication delays have been modelled as uniform randomly distributed time-varying delays with a maximum value of 0.2 second $[8,9,10,11,12,13]$. However, there are cases when communication delays are caused by an adversary, such as a cyberattacker, rather than being naturally occurring. For example, in [14] a Denial of Service (DoS) attack on a vehicle platoon could be shown as a communication delay between the vehicles. These artificial delays escape the uniform random assumptions that were made for natural delays. Comparatively, solutions from the transportation community have primarily focused on the spatial and temporal control of connected vehicles for intersection control [15] and reducing the inter-vehicular gaps between vehicles to improve traffic density $[16,17]$. Few studies appearing in the transportation community study the effects of communication delays on CAVNs [18, 19].

Different studies, as mentioned earlier, considered the effects of natural time-varying delay in autonomous vehicles and platoon stability in this context. In [8], a distributed adaptive collaborative control strategy that exploits information coming from connected vehicles to achieve leader synchronization is proposed to stabilize the platoon considering the Lyapunov-Krasovskii approach. In [9], a distributed control protocol that acts on every vehicle in the platoon was proposed, and the stability of the platoon is proven by using the Lyapunov-Razumikhin theorem. In [10], a distributed PID controller was designed to mitigate the unwanted effects caused by random time-varying communication delays on the leader following task.

In [11], convergence guarantees were found for a CAVN platoon using a consensus method for an arbitrary number of vehicles. Time delays in the communication links were once again random and time-varying, but it was assumed that by transmitting timestamps along with the vehicular states that the time delays were measurable. In [12], the effects of time-varying communication delays were studied in an elaborate system model that also modelled physical factors including wind speed, road slope, and rolling resistance. In the area of networked control systems, randomly varying long time-delays were considered, and an optimal controller was designed based on a linear-quadratic Gaussian control scheme [20].

Despite the progress made to address the effects of inter-vehicular communication delays, most studies are limited to addressing naturally occurring delays [21, 22]. The few, such as [14], that address adversarial communication delays however emphasize the detection and estimation of delays rather than designing controllers to mitigate them. Furthermore, there is no peer-reviewed literature that explores the parameter choice of time-varying delay functions (since few studies address adversarial delays to begin with, most studies assumed uniform randomly distributed delay functions, or in [23] time-varying delays with min-max bounds were considered). To accomplish this, the platoon dynamics model and the platoon's control goal (flow equilibrium) are proposed in Sect. 2. In CAVN literature, platooning vehicles are said to be in flow equilibrium if all vehicles 
in the platoon are equal distances apart from each other and travelling at the same velocity. It sometimes referred to as the Cooperative Adaptive Cruise Control problem or the longitudinal control of CAVN task. The proposed controller is able to stabilize the platoon from some space of initial conditions to the flow equilibrium. The following are the main contributions of the study:

- Study and verify the stability of the CAVN with constant and time-varying communication delays. This is essential to ensure the stability and safety of the future CAVN under timevarying communication delays.

- Development of an optimal velocity model with control gains space to stabilize CAVN with time-varying communication delays. This includes control gains charts with stable and unstable domains for the flow equilibrium.

- Perform simulation results of platoon stability with updating control gains and parameter choices for the periodic time-varying delay functions.

- Perform experimental results with four connected autonomous robots with the proposed control gains space to verify the stability charts with a real-time system considering constant and time-varying delays.

- Identify a specific region of instability caused by the periodic time-varying delay that could not be identified using the bounded time delay assumptions from previous studies.

Compared to [24], the proposed study considers the dynamics of CAVN with time delays under the assumption that the time lag depends on the distance between vehicles. It is assumed that the communication time delays among vehicles increase as the distance between the vehicles increases. The generalized eigenvalues are used to construct the maximal admissible delay region of stability of the model.

In order to guarantee the safety and mobility of the platoon, a control system that guarantees the existence of a unique flow equilibrium considering plant and string stability is proposed in Sects. 3, 4 and 5. In the constant delay case, the characteristic equation has an infinite number of zeros in the complex plane, where all of which should be considered during the stability analysis. A self-contained theoretical analysis to study the distribution of the characteristic roots in the complex plane and give sufficient conditions for stability is introduced.

In the time-varying delay case, stability conditions cannot be given as closed-form expressions of the system parameters because the corresponding monodromy operator is infinitely dimensional. However, the semi-discretization method is used to derive stability properties for the flow equilibrium. The method is an efficient numerical method based on a special kind of discretization technique with respect to the past effects only [25]. By employing this method, a Floquet transition matrix is defined, which is an approximation to the infinite-dimensional monodromy operator corresponding to the linear delayed system, to explore the stability of the flow equilibrium [26, 27]. Since the semi-discretization method preserves the stability for delay differential equations [28, 29], it is used to construct approximate stability charts in Sect. 6. These charts are diagrams constructed in the plane of the system parameters that show stable and unstable domains for the flow equilibrium. Additionally, numerical simulations are performed to verify the results in the analytical sections. 
Through the numerical examples, the updating control gains and parameter choices are observed for the periodic time-varying delay function that affects platoon stability. Sect. 7 presents a robotic test-bed and experimentally validating the conclusions about the CAVN platoon under periodic time-varying delays and large magnitudes of delays. Finally, Sect. 8 summarizes the results.

\section{The Model}

Consider $n+1$ vehicles in single-lane road and let $i=0,1, \ldots, n$. The index $i=0$ represents the leading vehicle and $n$ is the total number of the following vehicles. A control system model for a regular car platoon with communications delays of the following form is considered

$$
\left[\begin{array}{c}
\dot{s}_{i}(t) \\
\dot{v}_{i}(t)
\end{array}\right]=\left[\begin{array}{ll}
0 & 1 \\
0 & 0
\end{array}\right]\left[\begin{array}{c}
s_{i}(t) \\
v_{i}(t)
\end{array}\right]+\left[\begin{array}{l}
0 \\
1
\end{array}\right] u_{i}(t)
$$

where states $s_{i}$ and $v_{i}$ are the position and velocities, respectively, and $u_{i}$ is the control signal that drives the vehicle $i$ as considered in [30]. The output of the control signal can be written as

$$
u_{i}(t)=\sum_{j=0}^{i-1}\left[\alpha_{i, j}\left(\mathcal{V}\left(h_{i, j}\left(t-\epsilon_{i, j}(t)\right)\right)-v_{i}\left(t-\epsilon_{i, j}(t)\right)\right) \beta_{i, j}\left(v_{j}\left(t-\epsilon_{i, j}(t)\right)-v_{i}\left(t-\epsilon_{i, j}(t)\right)\right)\right],
$$

where $\alpha_{i, j} \in \mathbb{R}$ and $\beta_{i, j} \in \mathbb{R}$ are control gains, and $\epsilon_{i, j}(t)$ is a non-negative, bounded, and $T$-periodic function that represents the communication time delay for data transferred from vehicle $j \in\{0, \ldots, i-1\}$ to $i$, see Fig. 1 . The index $j=0,1, \ldots, i-1$ enumerates the vehicles in front of the vehicle $i$. The function $\mathcal{V}: \mathbb{R} \rightarrow \mathbb{R}$ is a range policy function that maps a distance to a velocity, and $h_{i, j}(t)$ represents the distance between the vehicles accounting for the length $l_{k}$ of the $k$-th vehicle

$$
h_{i, j}(t)=\frac{1}{i-j}\left(s_{j}(t)-s_{i}(t)-\sum_{k=j}^{i-1} l_{k}\right) .
$$

The used continuous and smooth range policy function is

$$
\mathcal{V}(h)= \begin{cases}0 & h \leq h_{\mathrm{st}} \\ \frac{v_{\max }}{2}\left[1-\cos \left(\frac{\pi\left(h-h_{\mathrm{st}}\right)}{h_{\text {go }}-h_{\mathrm{st}}}\right)\right] & h_{\mathrm{st}}<h<h_{\mathrm{go}}, \\ v_{\max } & h \geq h_{\mathrm{go}}\end{cases}
$$

where $h_{\mathrm{st}}, h_{g o}$ and $v_{\max }$ are positive constants. The function $\mathcal{V}$ implies that the vehicle tends to stop for small distances $h \leq h_{\text {st }}$ and aims to maintain the preset maximum speed $v_{\max }$ for large distances $h \geq h_{\mathrm{go}}$. In the middle range, $h_{\mathrm{st}}<h<h_{g o}$, the desired velocity is determined by the specific function given by cosine.

In the literature, platooning cars are called as uniform flow equilibrium when each car moves at a constant velocity of $v^{*}$ with constant headway $h^{*}$. In this scenario, it follows from (1) that

$$
s_{i}^{*}=v^{*} t-i h^{*}-\sum_{k=0}^{i-1} l_{k} \quad \text { and } \quad \mathcal{V}\left(h^{*}\right)=v^{*} .
$$

Notice that flow equilibrium is independent of time delays, the platoon size, and control gains. In this work, the plant stability and string stability of the linear system about the flow equilibrium are studied. 


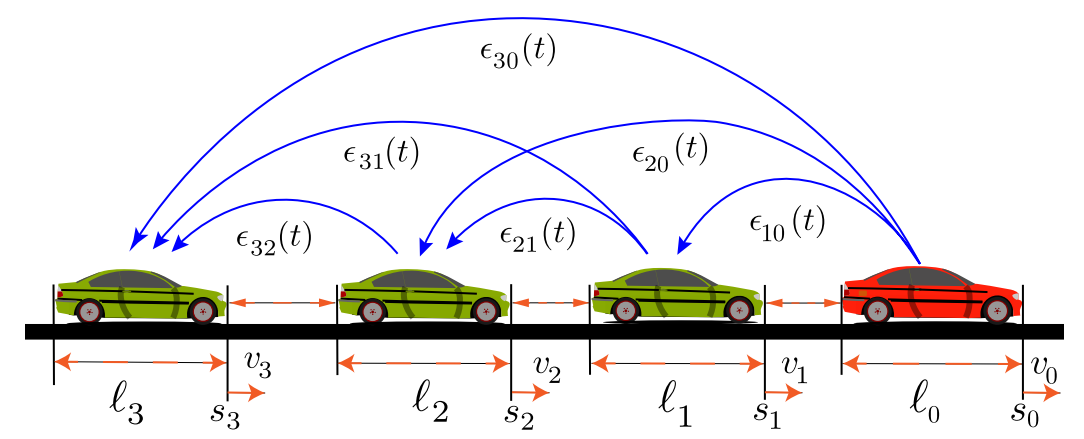

Figure 1: Schematic representation of vehicles in the model (1) with $n=3$. The car in red is the lead car (index 0). The blue arrows represent the flow of information which are subjected to delays $\epsilon_{i j}(t)$.

\section{Stability Analysis}

This section presents plant stability analysis of (1)-(4) when $\epsilon_{i j}(t)=\epsilon(t)$. A vehicle network is said to be plant stable when the leading vehicle moves with a constant speed, perturbations in the states of following vehicles approach to zero [31]. Let $\tilde{v}_{0} \equiv 0$ and seek $\tilde{v}_{i}(t) \rightarrow 0$ as $t \rightarrow \infty$ for $i=1, \ldots, n[31]$. This is equivalent to prove that the zero solution of the linearization of (1)-(4) about the flow equilibrium is stable.

To derive the linear system corresponding to the flow equilibrium, the perturbations about the flow equilibrium are defined as $\tilde{\mathbf{X}}_{i}=\left[\tilde{s}_{i}(t), \tilde{v}_{i}(t)\right]^{T}=\left[s_{i}(t), v_{i}(t)\right]^{T}-\left[s_{i}^{*}, v^{*}\right]^{T}$. Then, substituting $\tilde{\mathbf{X}}_{i}$ in (1) leads to

$$
\dot{\tilde{\mathbf{X}}}_{i}(t)=\mathbf{A}_{0} \tilde{\mathbf{X}}_{i}(t)+\sum_{j=0}^{i-1}\left(\mathbf{A}_{i, j} \tilde{\mathbf{X}}_{i}\left(t-\epsilon_{i, j}(t)\right)+\mathbf{B}_{i, j} \tilde{\mathbf{X}}_{j}\left(t-\epsilon_{i, j}(t)\right),\right.
$$

where

$$
\mathbf{A}_{0}=\left[\begin{array}{ll}
0 & 1 \\
0 & 0
\end{array}\right], \mathbf{A}_{i, j}=-\left[\begin{array}{cc}
0 & 0 \\
\psi_{i, j} & \kappa_{i, j}
\end{array}\right], \mathbf{B}_{i, j}=\left[\begin{array}{cc}
0 & 0 \\
\psi_{i, j} & \beta_{i, j}
\end{array}\right]
$$

with $\kappa_{i, j}=\alpha_{i, j}+\beta_{i, j}$ and

$$
\psi_{i, j}=\frac{\alpha_{i, j} \mathcal{V}^{\prime}\left(h^{*}\right)}{i-j} .
$$

Here $\mathcal{V}^{\prime}$ is the first derivative of the range policy $\mathcal{V}$ with respect to $h$.

Since the delay in the system (6) is a time-periodic function, the stability of the zero solution of (6) is determined by the location of the system Floquet multipliers, which are the nonzero elements of the spectrum of the system monodromy matrix. When all the Floquet multipliers are within the unit circle of the complex plane, the zero solution is stable. When one or more of the Floquet multipliers are on the unit circle and the rest of them are inside the unit circle, the zero solution becomes unstable [32]. The monodromy matrix of the system (6) is an infinite-dimensional operator and has no closed-form due to time-periodic delay. Hence, the semi-discretization method [26, 27] is used to approximate it by a finite-dimensional matrix based on a special kind of discretization technique with respect to the past effects only [25]. 
Let $\mathbf{X}(t)=\left[\tilde{\mathbf{X}}_{1}(t), \cdots, \tilde{\mathbf{X}}_{n}(t)\right]^{T}$. When the delay functions are the same, that is, $\epsilon_{i j}(t)=\epsilon(t)$, system (6) can be written as

$$
\dot{\mathbf{X}}(t)=\mathbf{H}_{1} \mathbf{X}(t)+\mathbf{H}_{2} \mathbf{X}(t-\epsilon(t))
$$

where

$$
\mathbf{H}_{1}=\operatorname{diag}\left(\mathbf{A}_{0}, \mathbf{A}_{0}, \ldots, \mathbf{A}_{0}\right)
$$

and

$$
\begin{aligned}
\mathbf{H}_{2} & =\operatorname{diag}\left(0, \mathbf{A}_{2,1}, \mathbf{A}_{3,1}+\mathbf{A}_{3,2} \ldots, \sum_{j=1}^{n-1} \mathbf{A}_{n, j}\right) \\
& +\left[\begin{array}{ccccc}
\mathbf{0} & \mathbf{0} & \mathbf{0} & \cdots & \mathbf{0} \\
\mathbf{B}_{2,1} & \mathbf{0} & \mathbf{0} & \cdots & \mathbf{0} \\
\mathbf{B}_{3,1} & \mathbf{B}_{3,2} & \mathbf{0} & \cdots & \mathbf{0} \\
\vdots & \vdots & \vdots & \ddots & \vdots \\
\mathbf{B}_{n, 1} & \mathbf{B}_{n, 2} & \cdots & \mathbf{B}_{n, n-1} & \mathbf{0}
\end{array}\right]
\end{aligned}
$$

Denote $\epsilon_{\max }:=\max _{t \in[0, T]}\{\epsilon(t)\}$ and $\mathcal{C}=C\left(\left[-\epsilon_{\max }, 0\right]\right.$. For any history function $\phi \in \mathcal{C}$, the solution operator is defined as $U: \mathcal{C} \rightarrow \mathcal{C}$ by $X(t)=U(t) \phi$. Since (8) is $T$-periodic, the monodromy matrix can be defined by setting $t=T$, that is,

$$
X(T)=U(T) \phi
$$

In the following, the semi-discretization method is used to approximate the infinite dimensional monodromy matrix $U(T)$ by a finite dimensional matrix $[27,26]$.

Suppose the time period $T$ is divided into $K$ equal intervals of length $h=T / K$. Let $t_{m}=m h$. Then, for $t \in\left[t_{m}, t_{m+1}\right]$, the time delay is approximated as [26]

$$
\epsilon_{m}=\frac{1}{h} \int_{t_{m}}^{t_{m+1}} \epsilon(t) d t .
$$

Following [26], in each discretization interval $\left[t_{m}, t_{m+1}\right)$, the delayed state can be approximated as

$$
\begin{aligned}
\mathbf{X}(t-\epsilon(t)) & \approx \mathbf{X}\left(t-\epsilon_{m}\right) \\
& \approx r_{m, 0}(t) \mathbf{X}\left(t_{m-l_{m}}\right)+r_{m, 1}(t) \mathbf{X}\left(t_{m-l_{m}+1}\right),
\end{aligned}
$$

where $l_{m}=\operatorname{int}\left(\frac{\epsilon_{m}}{h}+\frac{1}{2}\right)$ with int denoting the integer-part function, and

$$
r_{m, 0}(t)=\frac{\epsilon_{m}+\left(m-l_{m}+1\right) h-t}{h}
$$

and

$$
r_{m, 1}(t)=\frac{t-\left(m-l_{m}\right) h-\epsilon_{m}}{h}
$$

The scheme of the approximation in (11) is shown in Fig. 2 and more details are provided in [26, Chapter 3]. Hence, for $t \in\left[t_{m}, t_{m+1}\right]$ and $m=0, \ldots, K-1$, system (8) can be approximated by an autonomous system of ordinary differential equations as

$$
\dot{\mathbf{X}}(t)=\mathbf{H}_{1} \mathbf{X}(t)+\mathbf{H}_{2}\left(r_{m, 0}(t) \mathbf{X}\left(t_{m-l_{m}}\right)+r_{m, 1}(t) \mathbf{X}\left(t_{m-l_{m}+1}\right)\right), \quad t \in\left[t_{m}, t_{m+1}\right] .
$$


By using the variation of constants formula, the general solution of (12) can be written as

$$
\mathbf{X}(t)=\mathrm{e}^{\mathbf{H}_{1}\left(t-t_{m}\right)} \mathbf{X}\left(t_{m}\right)+\int_{t_{m}}^{t}\left\{\mathrm{e}^{\mathbf{H}_{1}(t-s)} \mathbf{H}_{2}\left(r_{m, 0}(s) \mathbf{X}\left(t_{m-l_{m}}\right)+r_{m, 1}(s) \mathbf{X}\left(t_{m-l_{m}+1}\right)\right)\right\} d s .
$$

Using the notation $\mathbf{X}_{m}:=\mathbf{X}\left(t_{m}\right)$, with $t=t_{m+1}$, the solution over one discrete step can be formulated as

$$
\mathbf{X}_{m+1}=\mathbf{P} \mathbf{X}_{m}+\mathbf{R}_{m, 0} \mathbf{X}\left(t_{m-l_{m}}\right)+\mathbf{R}_{m, 1} \mathbf{X}\left(t_{m-l_{m}+1}\right)
$$

where $\mathbf{P}=\mathrm{e}^{\mathbf{H}_{1} h}$, then

$$
\mathbf{R}_{m, 0}=\left(\int_{0}^{h} \frac{\epsilon_{m}-\left(l_{m}-1\right) h-s}{h} e^{\mathbf{H}_{1}(h-s)} d s\right) \mathbf{H}_{2}
$$

and

$$
\mathbf{R}_{m, 1}=\left(\int_{0}^{h} \frac{s+l_{m} h-\epsilon_{m}}{h} e^{\mathbf{H}_{1}(h-s)} d s\right) \mathbf{H}_{2} .
$$

Define the augmented state vector as

$$
\mathbf{Z}_{m+1}=\left[\mathbf{X}_{m}, \mathbf{X}_{m-1}, \ldots, \mathbf{X}_{m-l_{\max }}\right]^{T}
$$

with $l_{\max }=\max \left\{l_{m}\right\}$. The linear discrete map is

$$
\mathbf{Z}_{m+1}=\mathbf{D}_{m} \mathbf{Z}_{m}
$$

where $\mathbf{D}_{m}$ is given by

$$
\mathbf{D}_{m}=\left[\begin{array}{c|ccccc}
\mathbf{P} & \mathbf{0} & \cdots & \mathbf{0} & \mathbf{0} & \mathbf{0} \\
\hline \mathbf{I}_{2 n} & \mathbf{0} & \cdots & \mathbf{0} & \mathbf{0} & \mathbf{0} \\
\mathbf{0} & \mathbf{I}_{2 n} & \ddots & \mathbf{0} & \mathbf{0} & \mathbf{0} \\
\vdots & \vdots & \ddots & \ddots & \vdots & \vdots \\
\mathbf{0} & \mathbf{0} & \cdots & \mathbf{I}_{2 n} & \mathbf{0} & \mathbf{0} \\
\mathbf{0} & \mathbf{0} & \cdots & \mathbf{0} & \mathbf{I}_{2 n} & \mathbf{0}
\end{array}\right]+\left[\begin{array}{c|ccccccc}
\mathbf{0} & \mathbf{0} & \cdots & \mathbf{R}_{m, 1} & \mathbf{R}_{m, 0} & \cdots & \mathbf{0} \\
\hline \mathbf{0} & \mathbf{0} & \cdots & 0 & \mathbf{0} & \cdots & \mathbf{0} \\
\mathbf{0} & \mathbf{0} & \cdots & \mathbf{0} & \mathbf{0} & \cdots & \mathbf{0} \\
\vdots & \mathbf{0} & & \vdots & \vdots & \cdots & \mathbf{0} \\
\mathbf{0} & \mathbf{0} & \cdots & \mathbf{0} & \mathbf{0} & \cdots & \mathbf{0} \\
\mathbf{0} & \mathbf{0} & \cdots & \mathbf{0} & \mathbf{0} & \cdots & \mathbf{0}
\end{array}\right]
$$

In (17), the matrices $\mathbf{R}_{m, 1}$ and $\mathbf{R}_{m, 0}$ are located at the $\left(l_{m}-1\right)$-th and $l_{m}$-th places within the right upper block of $\mathbf{D}_{m}$.

Applying the discrete map (16) over the period $T=K h$ leads to

$$
\mathbf{Z}_{m+1}=\Phi \mathbf{Z}_{0},
$$

where the transition matrix is defined, as

$$
\Phi=\mathbf{D}_{K-1} \cdots \mathbf{D}_{1} \mathbf{D}_{0}
$$

This represents a finite-dimensional approximation of the infinite dimensional monodromy matrix $U(T)$. If all the eigenvalues of $\Phi$ are within the unit circle, then the zero solution is stable, and hence, system (6) is plant stable. 


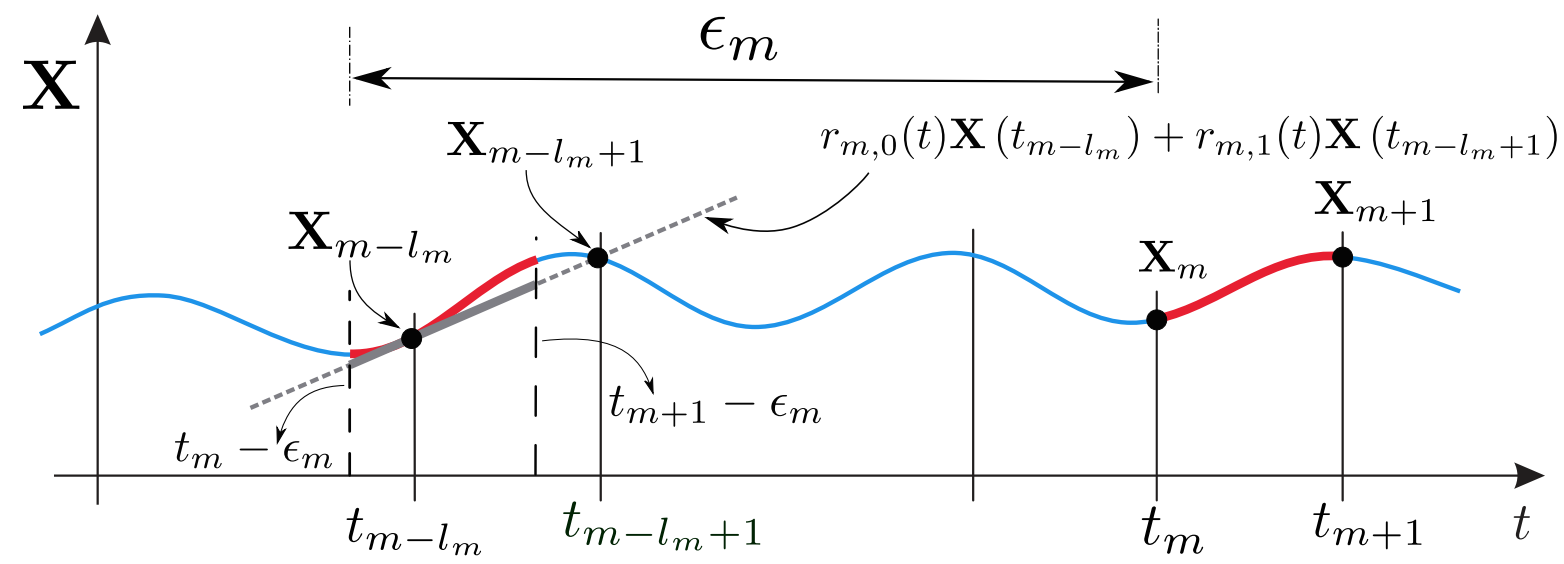

Figure 2: The figure shows approximation of the delayed term $\mathbf{X}\left(t-\epsilon_{m}\right)$ (the gray dashed line), where $\mathbf{X}_{\mathrm{i}}=\mathbf{X}\left(t_{\mathrm{i}}\right)$.

\section{Critical time delay}

This section drives the critical time delay and characterizes the maximal admissible delay region such that the time-delayed system (6) remains plant stable with constant time delay $\epsilon(t) \equiv \epsilon:=\epsilon_{\text {avg }}$, where $\epsilon_{\mathrm{avg}}$ is the average value of the time delay function

$$
\epsilon_{\mathrm{avg}}=\frac{1}{T} \int_{0}^{T} \epsilon(t) d t
$$

where $T$ is the period of $\epsilon(t)$. In this case, (6) can be written as

$$
\dot{\tilde{\mathbf{X}}}_{i}(t)=\mathbf{A}_{0} \tilde{\mathbf{X}}_{i}(t)+\sum_{j=0}^{i-1}\left(\mathbf{A}_{i, j} \tilde{\mathbf{X}}_{i}(t-\epsilon)+\mathbf{B}_{i, j} \tilde{\mathbf{X}}_{j}(t-\epsilon),\right.
$$

for $i=1, \ldots, n$. In the case of constant delay, the zero solution in (21) is stable if the real part of all eigenvalues of the characteristic equation is negative.

Recall that $\mathbf{X}(t)=\left[\tilde{\mathbf{X}}_{1}(t), \cdots, \tilde{\mathbf{X}}_{n}(t)\right]^{T}$. To find the characteristic equation, let

$$
\mathbf{X}(t)=\mathbf{q} \mathrm{e}^{\lambda t}, \lambda \in \mathbb{R}, \mathbf{q} \in \mathbb{R}^{n} .
$$

Then, the characteristic equation associated with (21) has the form

$$
\Delta(\lambda)=\prod_{i=1}^{n} \Delta_{i}(\lambda)=0
$$

with

$$
\Delta_{i}(\lambda)=\lambda^{2}+\sum_{j=1}^{i-1} \kappa_{i j} e^{-\lambda \epsilon} \lambda+\sum_{j=1}^{i-1} \psi_{i j} e^{-\lambda \epsilon} .
$$

Thus, $\Delta(\lambda)$ has a root if and only if $\Delta_{i}(\lambda)=0$ for some $i \in\{1,2, \ldots, n\}$. Let

$$
a_{i}=\sum_{j=1}^{i-1} \kappa_{i j} \text { and } b_{i}=\sum_{j=1}^{i-1} \psi_{i j}
$$


It is clear when $b_{i}<0$ for some $i \in\{1, \ldots, n\}, \Delta(\lambda)$ has a positive real root, and hence, the zero solution is unstable. Hence, for stability, $b_{i}>0$ for all $i \in\{1, \ldots, n\}$.

To construct the maximum range of time delay for stability, first, the stability condition when $\epsilon=0$ is considered. Then, by the Routh-Hurwitz stability criterion, the following can be obtained.

Lemma 4.1. When $\epsilon=0$, the zero solution of (6) is stable if and only if, for all $i \in\{1, \ldots, n\}$,

$$
a_{i}>0 \text { and } b_{i}>0 .
$$

Let $\epsilon>0$ and suppose $\lambda_{i}=I \eta_{i}\left(\eta_{i}>0 I=\sqrt{-1}\right)$ is a purely imaginary eigenvalue of $\Delta_{i}(\lambda)=0$. By separating the real and imaginary parts of $\Delta_{i}(\lambda)=0$, the following can be obtained:

$$
\begin{aligned}
\eta_{i}^{2} & =a_{i} \eta_{i} \sin \left(\eta_{i} \epsilon\right)+b_{i} \cos \left(\eta_{i} \epsilon\right), \\
0 & =a_{i} \eta_{i} \cos \left(\eta_{i} \epsilon\right)-b_{i} \sin \left(\eta_{i} \epsilon\right) .
\end{aligned}
$$

Squaring and adding the above equations lead to

$$
\Xi_{i}\left(s_{i}\right):=s_{i}^{2}-a_{i}^{2} s_{i}-b_{i}^{2}=0,
$$

where $s_{i}=\eta_{i}^{2}$. The quadratic polynomial $\Xi_{i}$ has exactly one positive root

$$
\eta_{i, 0}=\left(\frac{a_{i}^{2}+\sqrt{a_{i}^{4}+4 b_{i}^{2}}}{2}\right)^{\frac{1}{2}} .
$$

Plugging $\eta_{i}=\eta_{i, 0}$ into (27)-(28) and solving the resulting equation for $\cos \left(\eta_{i, 0} \epsilon\right)$ and $\sin \left(\eta_{i, 0} \epsilon\right)$ leads to

$$
\left\{\begin{aligned}
\cos \left(\eta_{i, 0} \epsilon\right) & =\frac{b_{i} \eta_{i, 0}^{2}}{a_{i}^{2} \eta_{i, 0}^{2}+b_{i}^{2}}:=P_{0}, \\
\sin \left(\eta_{i, 0} \epsilon\right) & =\frac{a_{i} \eta_{i, 0}^{3}}{a_{i}^{2} \eta_{i, 0}^{2}+b_{i}^{2}}:=Q_{0} .
\end{aligned}\right.
$$

Hence, the sequence of solution with respect to $\epsilon$

$$
\epsilon_{i, k}=\frac{1}{\eta_{i, 0}} \arccos \left(\frac{b_{i} \eta_{i, 0}^{2}}{a_{i}^{2} \eta_{i, 0}^{2}+b_{i}^{2}}\right)+\frac{2 k \pi}{\eta_{i, 0}}, k \in \mathbb{Z}_{0}^{+}
$$

when $a_{i}>0$ and

$$
\epsilon_{i, k}=\frac{1}{\eta_{i, 0}}\left[2 \pi-\arccos \left(\frac{b_{i} \eta_{i, 0}^{2}}{a_{i}^{2} \eta_{i, 0}^{2}+b_{i}^{2}}\right)\right]+\frac{2 k \pi}{\eta_{i, 0}}, k \in \mathbb{Z}_{0}^{+}
$$

when $a_{i}<0$. Here, $\mathbb{Z}_{0}^{+}=\{0,1, \ldots\}$. Taking the derivative of $\Delta_{i}(\lambda(\epsilon))=0$ with respect to $\epsilon$ and using (27) lead to

$$
\left.\operatorname{Re}\left(\frac{d \lambda(\epsilon)}{d \epsilon}\right)^{-1}\right|_{\epsilon=\epsilon_{i, k}}=\frac{a_{i}^{2}+2 \eta_{i}^{2}}{a_{i}^{2} \eta_{i}^{2}+b_{i}^{2}}>0 .
$$

Hence, $\lambda(\epsilon)$ crosses the imaginary axis from left to right in a neighbourhood of $\epsilon=\epsilon_{i, k}$.

Theorem 4.1. Assume (26) is satisfied such that the zero solution of (6) is stable when $\epsilon=0$. Then, the zero solution remains stable for $\epsilon \in\left(0, \epsilon^{*}\right)$; and becomes unstable for $\epsilon>\epsilon^{*}$ where

$$
\epsilon^{*}=\min \left\{\epsilon_{i, 0}: i \in\{1, \ldots, n\}\right\} .
$$

Theorem 4.1 implies that all eigenvalues of $(23)$ are negative when $\epsilon \in\left(0, \epsilon^{\star}\right)$, and hence, the $\tilde{v}(t)$ of $(6)$ goes to zero as $t \rightarrow \infty$, that is, the system (21) is plant stable. 


\section{$5 \quad$ String Stability}

In this section, the string stability of (21) is studied. This is critical in the dynamics of the vehicle network to avoid amplifying spacing errors downstream the traffic flow [33]. The string instability of a platoon can cause the emergence of a jam, such as stop and go, in circuits and single-lane roads. Mathematically, a vehicle with transfer function $L(\cdot)$ is string stable if and only if $|L(j \omega)| \leq 1$ for all $\omega \in \mathbb{R}^{+}$. Through this section, it is assumed that $(21)$ is a plant stable $\left(\epsilon \in\left(0, \epsilon^{*}\right)\right)$.

The velocity $\tilde{v}_{i}(t)$ in $(21)$ can be written $\tilde{v}_{i}(t)=\mathbf{c} \tilde{\mathbf{X}}_{i}(t)$ with $\mathbf{c}=[0,1]$. Let $V_{i}(s)$ be the Laplace transform of $v_{i}(t)$ and assume zero initial condition. Then, from (21) it can be obtained

$$
V_{i}(s)=\sum_{j=0}^{i-1} G_{i, j}(s) V_{j}(s)
$$

where

$$
G_{i, j}(s)=\mathrm{e}^{-\epsilon_{i} s} \mathbf{c} \times\left(s \mathbf{I}_{2}-\mathbf{A}_{0}-\mathrm{e}^{-\epsilon s} \sum_{j=0}^{i-1} \mathbf{A}_{i, j}\right)^{-1} \mathbf{B}_{i, j} \mathbf{E}(s) .
$$

Here $\mathbf{I}_{2}$ is the identity matrix of size 2 and $\mathbf{E}(s)=\left[s^{-1}, 1\right]^{T}$ that satisfies $\mathbf{Y}_{i}(s)=\mathbf{E}(s) V_{i}(s)$ where $\mathbf{Y}_{i}(s)$ is the Laplace transform of the state $\tilde{\mathbf{X}}_{i}(t)$ in $(21)$. The function $G_{i, j}$ is called the link transfer function, which acts as a dynamic weight along the link between vehicles $i$ and $j$ [34]. Consequently, by substituting $(7)$ in $(36), G_{i, j}$ can be written as

$$
G_{i, j}(s)=\frac{\left(s \beta_{i, j}+\psi_{i, j}\right) \mathrm{e}^{-\epsilon s}}{s^{2}+\sum_{k=0}^{i-1}\left(s \kappa_{i, j}+\psi_{i, k}\right) \mathrm{e}^{-\epsilon s}} .
$$

To describe the dynamic relationship between any two vehicles $i$ and $j$, the transfer function $T_{i, j}$ from (35) can be expressed as

$$
V_{i}(s)=T_{i, j}(s) V_{j}(s)
$$

which contains the dynamics of all vehicles between vehicles $i$ and $j$. Because of the same connection topology in $(1)$, the string stability can be obtained with $T_{n, 0}(s)$, that is, the system is string stable if and only if

$$
\sup _{\omega>0}\left|T_{n, 0}(I \omega)\right|<1
$$

To find $T_{n, 0}(s)$, define

$$
\mathbf{G}(s):=\left[G_{i+1, j+1}(s)\right]_{j=0, \ldots, i-1}^{i=0, \ldots, n},
$$

where $\mathbf{G}(s) \in \mathbb{C}^{(n+1) \times(n+1)}$. Then, $T_{n, 0}(s)$ is given by the bottom-left elements of the matrix as

$$
\mathbf{T}(s)=\sum_{k=1}^{n}(\mathbf{G}(s))^{k}
$$

For the details on the mathematical derivation, please see for example [35]. 


\section{$6 \quad$ Numerical Results}

This section verifies the theoretical results with $n=3$ in Sects 3-5. The package SemiDiscretizationMethod.jl on Julia is employed to implement the semi-discretization method in Sect. 3. The following time-varying sinusoidal function is used

$$
\epsilon(t)=a-b(1-\cos (\omega t+c)),
$$

with the same default range policy described in (4) under $h_{\mathrm{st}}=0.1 m, h_{\mathrm{go}}=2.2 m, h^{*}=1$ and $v_{\max }=0.25 \mathrm{~m} / \mathrm{s}$.

The control gains $\alpha=0.3$ and $\beta=0.27$ with fixed $a=1$ are considered. First, $c=0$ is used and the amplitude $(b)$ and frequency $(\omega)$ vary. The boundary between stable and unstable regions in the parameter space of $b$ and $\omega$ are shown in Fig. 3a. A value from the stability/instability region is chosen, and then the eigenvalues of $\Phi$ are plotted in Figs. 4-5. As discussed in Sect. 3, when all eigenvalues of $\Phi$ in (19) lie inside the unit circle, speed error $\tilde{v}_{i}(t)$ approaches to zero, that is, system (6) is plant stable.

Then use $\omega=1$ and vary the parameter $c$. The boundary between stable and unstable regions in the parameter space of $b$ and $c$ is shown in Fig. 3b. When $b$ is fixed, the increase in $c$ does not affect the stability, while when $b$ increases with fixed $c$, the flow equilibrium losses the stability with $b>0.1$.

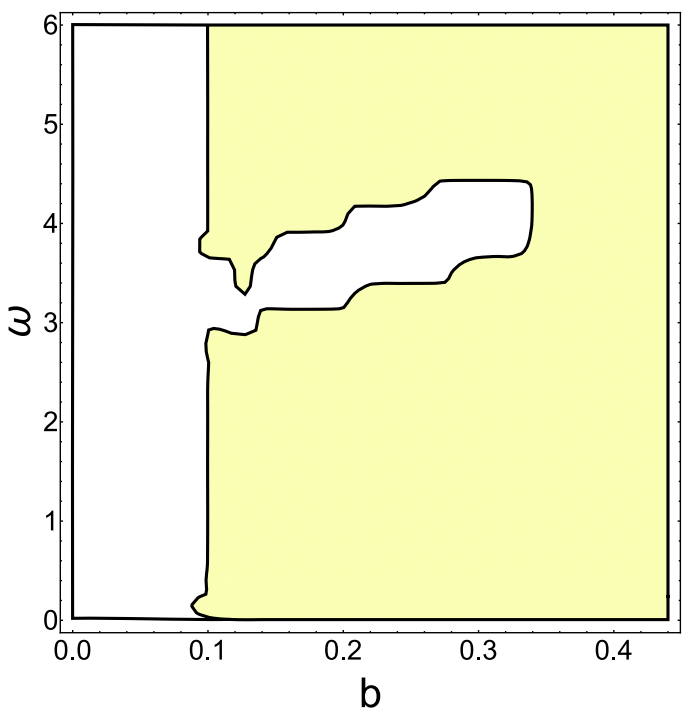

(a) bw-plane when $c=0$.

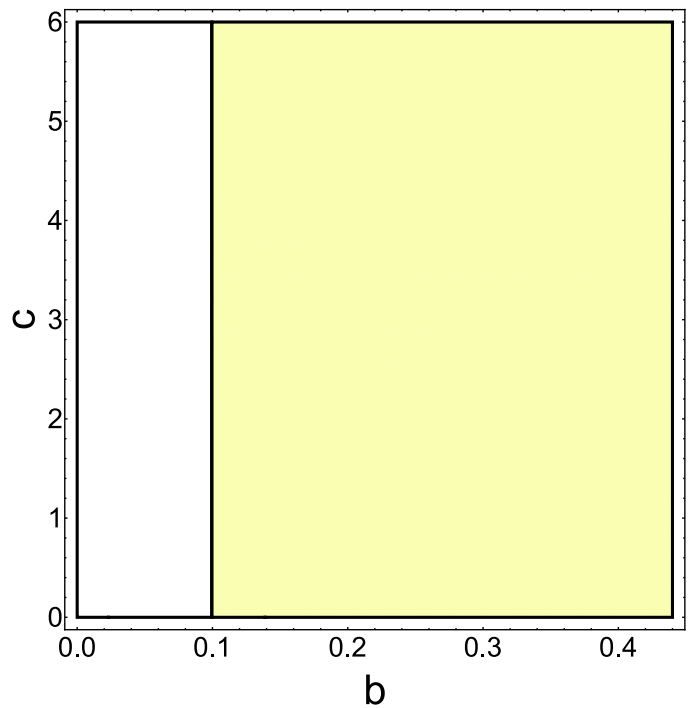

(b) $b c$-plane when $\omega=1$.

Figure 3: Stability chart for plant stability that is obtained by semi-discretization method for $h=0.1$ with $\epsilon(t)=a-b(1-\cos (\omega t+c))$. The stable region is shaded, and the unstable region is not shaded. The parameters $a=1, \alpha=0.3$ and, $\beta=0.27$ are fixed.

To compare between stability regions in system (6) and (21), with time-varying delay and constant delay, consider $\epsilon(t)=1-0.45(1-\cos (t))$ with its maximum value of $\epsilon_{\max }:=\max \{\epsilon(t)\}=1$. Fig. 6 shows the the boundary between stable and unstable regions in the parameter space of $\alpha$ and $\beta$ corresponding to $\epsilon(t)$ and $\epsilon_{\max }$. It can be observed that the time-varying delay has a larger stability margin than constant delay, although the stability boundaries for the two cases are not 

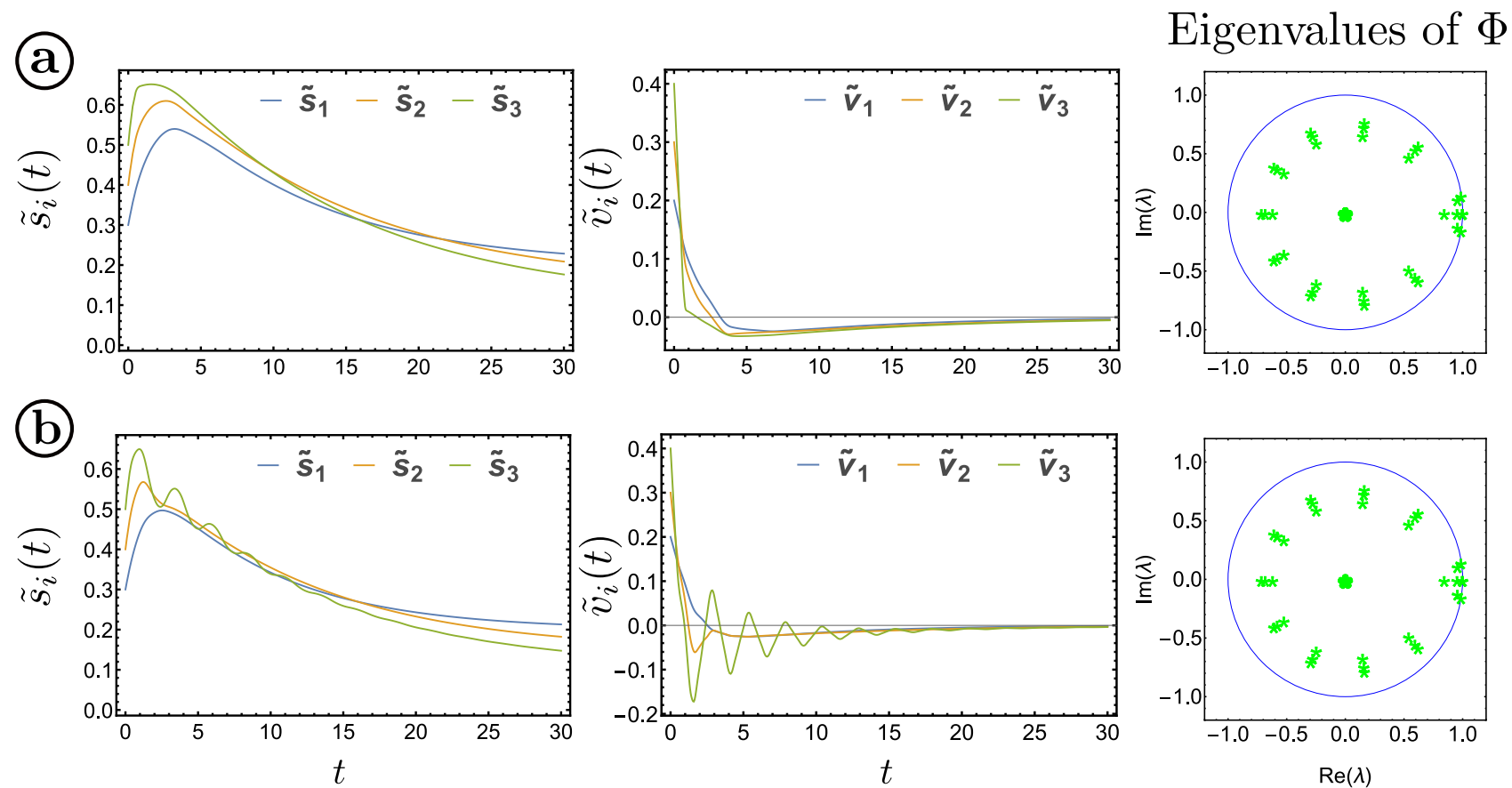

Figure 4: Time series of $\tilde{s}_{i}(t)$ and $\tilde{v}_{i}(t)$ of system (6) with (a) $b=0.4$ and $\omega=4.8$ (b) $b=0.2$ and $\omega=5$. The other parameter values are similar to those in Fig. 3a. Since all eigenvalues of $\Phi$ in (19) lie inside the unit circle, speed error $\tilde{v}_{i}(t)$ approaches to zero. Hence, system (6) is plant stable.
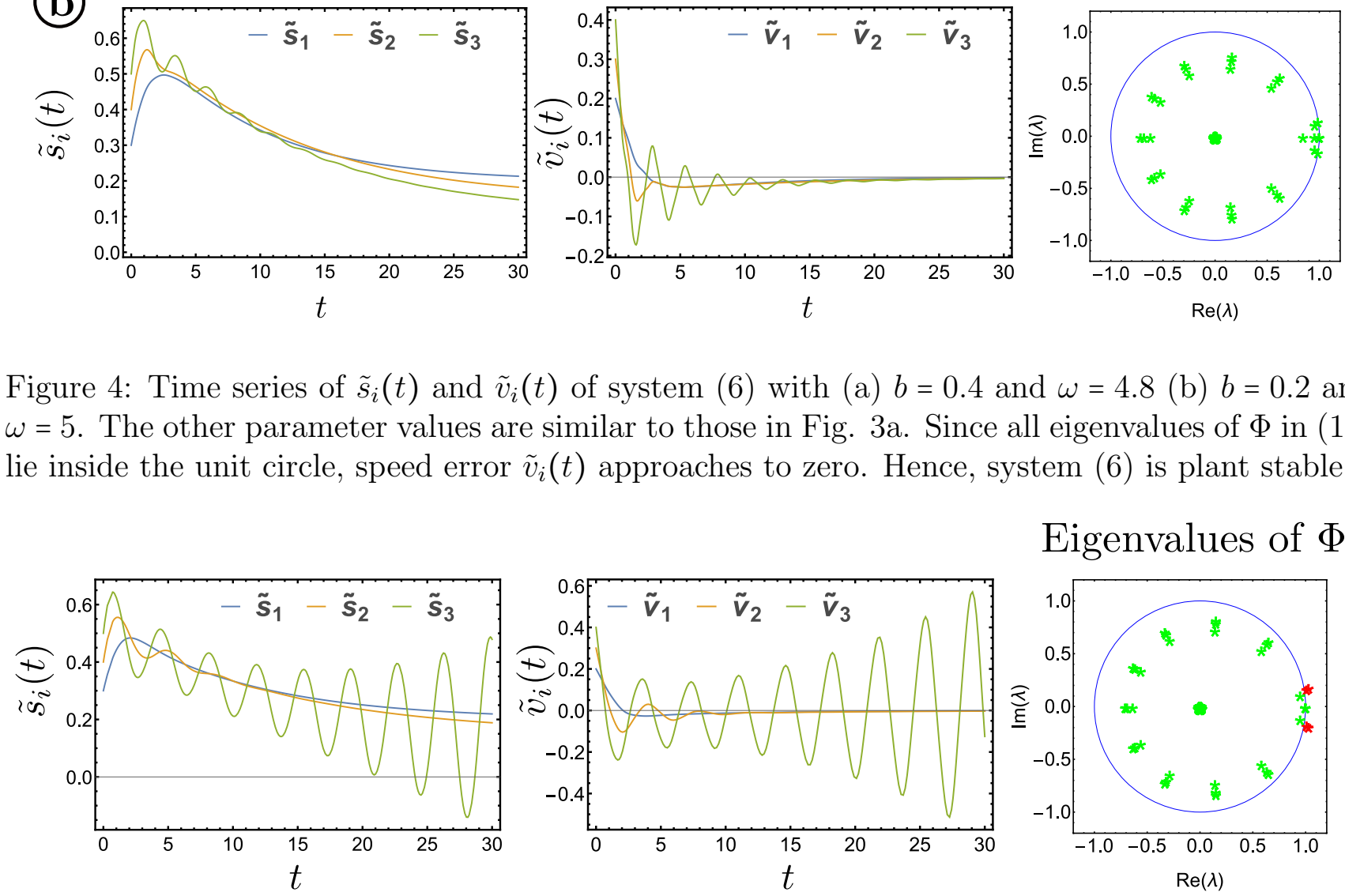

Eigenvalues of $\Phi$

Figure 5: Time series of $\tilde{s}_{i}(t)$ and $\tilde{v}_{i}(t)$ of system (6) with $b=0.15$ and $\omega=3.5$. System (6) is plant unstable because there exists an eigenvalue of $\Phi$ in (19) that lies outside the unit circle. Notice that the speed error $\tilde{v}_{i}(t)$ does not converge to zero.

far apart. The pair $(\alpha, \beta)=(0.25,0.31)$ is selected for further investigation and find that the flow equilibrium is stable with time-varying delays $\epsilon(t)$, see Fig. 7, while it is unstable with constant delay $\epsilon_{\max }$, see Fig. 7 .

Although all time delay functions in this section has a maximum value of 1 , stable and unstable dynamics appear based on the choice of the parameters of the time delay function as shown in Fig. 4, system (6). Consequently, the average value $\epsilon_{\text {avg }}$ of the time delay function defined in (20) 


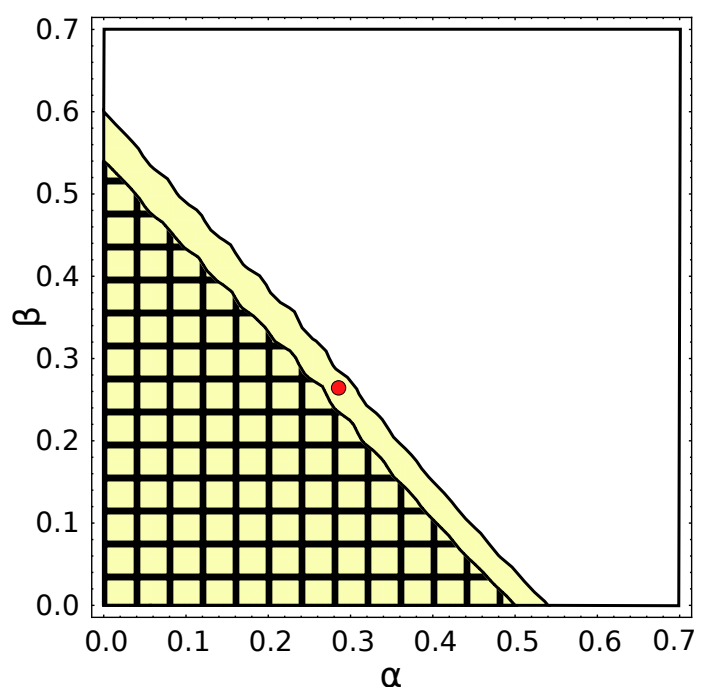

Figure 6: Stability chart for plant stability obtained by semi-discretization method for $h=0.1$ with time-varying delay $\epsilon(t)=1-0.45(1-\cos (t))$, where the stable region is shaded, and unstable region is not shaded. The cross-hatch pattern region presents the constant delay of $\epsilon_{\max }=1$. The time-varying delay has a slightly larger stability margin than constant delay.
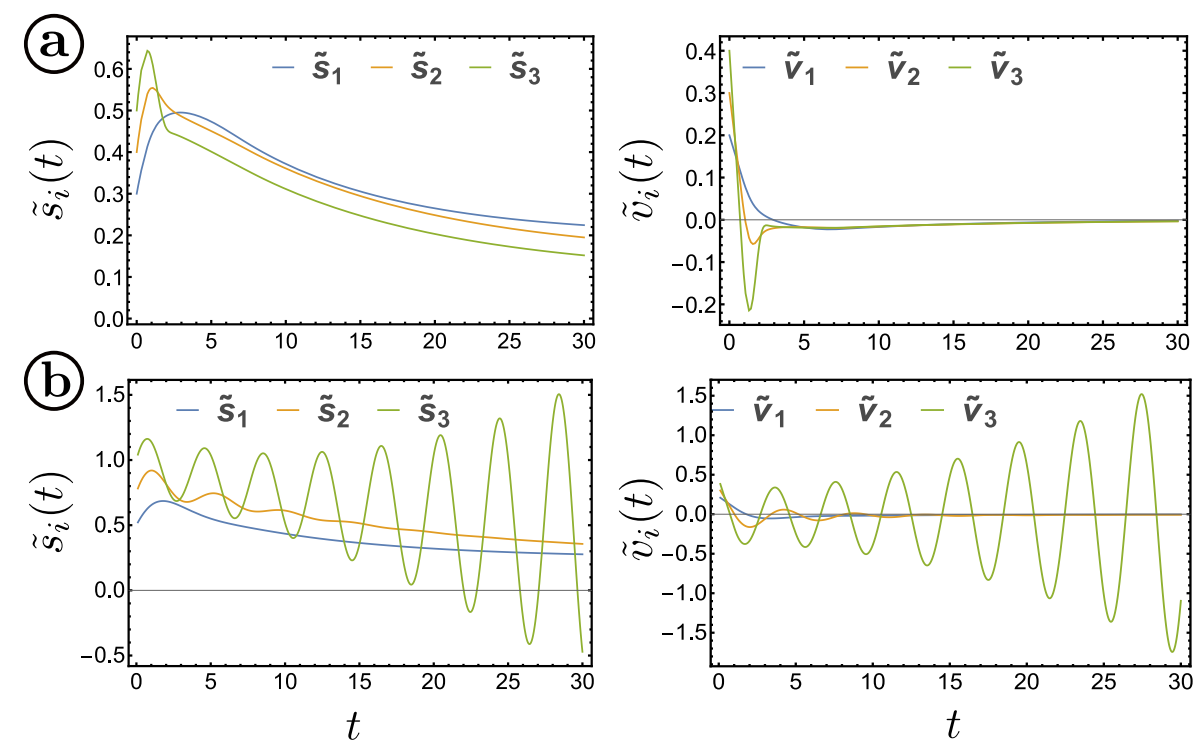

Figure 7: Time series of $\tilde{s}_{i}(t)$ and $\tilde{v}_{i}(t)$ of (a) System (6) with $\epsilon(t)=1-0.45(1-\cos (t))$ (b) System (21) with constant delay $\epsilon_{\max }:=\max \{\epsilon(t)\}=1$. The control gains $\alpha=0.25$ and $\beta=0.31$ corresponded to the red dot in Fig. 6.

is considered to study the string stability. It follows from (41) that the transfer function

$$
T_{3,0}(s)=G_{3,0}(s)+G_{2,0}(s) G_{3,2}(s)+G_{1,0}(s)\left(G_{3,1}(s)+G_{2,1}(s) G_{3,2}(s)\right)
$$

where $G_{i, j}$ is given by (36) with $\epsilon=\epsilon_{\text {avg. }}$. Straightforward calculations give $\epsilon_{\text {avg }}^{4 a}=0.6$ and $\epsilon_{\text {avg }}^{4 b}=0.8$. In fact, when the time delay is constant, the critical time delay given in (34) for system (21) is 
$\epsilon^{*}=0.898$, and hence, system $(21)$ is plant stable when $\epsilon=\epsilon_{\text {avg }}^{4 a}$ and $\epsilon=\epsilon_{\text {avg }}^{4 b}$. Fig. 8 shows $\left|T_{3,0}(I \omega)\right|$ when $\epsilon=\epsilon_{\mathrm{avg}}^{4 a} \backslash \epsilon_{\mathrm{avg}}^{4 b}$ with $5 \%$ deviation from the value of $\epsilon$. It can be observed that system (6) is string stable when $\epsilon=\epsilon_{\text {avg }}^{4 a}$ corresponding to Figs. 4a and string unstable with $\epsilon=\epsilon_{\mathrm{avg}}^{4 b}$ from time delay function in Fig. 4b.
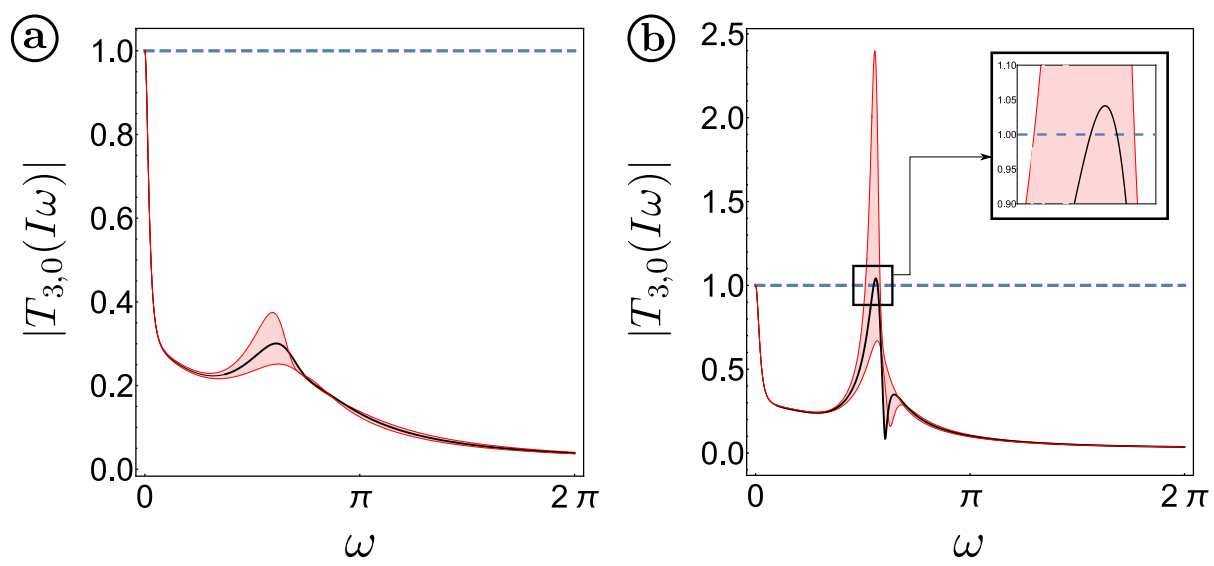

Figure 8: The curve of $\left|T_{3,0}(I \omega)\right|$ with $\epsilon=\epsilon_{\text {avg }}$ in Fig. 4 (a) $\epsilon_{\text {avg }}^{4 a}=0.6$ in Fig. $4 \mathrm{a}$ (b) $\epsilon_{\text {avg }}^{4 b}=0.8$ in Fig. 4b. The red colour represents the $5 \%$ deviation from the value of $\epsilon$. System (6) is string stable in (a) because the the maximum value of $\left|T_{3,0}(I \omega)\right|$ is less than 1 for all $\omega>0$ and it is string unstable in (b) since the supremum of $\left|T_{3,0}(I \omega)\right|$ is bigger than 1 for $\omega \in(0, \infty)$.

\section{Experiments}

In this section, the controller in (2) is used with four QBot2e robots for the physical experimentation. To briefly elaborate on the physical setup, each QBot2e robot is driven by the 2-wheeled Kobuki robot base and comes equipped with a Microsoft Kinect sensor, a gyroscope, wheel odometers, and a Raspberry Pi 3 Model B+ (ARM Cortex-A53 1.4GHz CPU, 1.0 GB memory, Raspbian operating system). The Raspberry $\mathrm{Pi}$ act as the onboard computing platform for each robot, and it runs a set of $\mathrm{C} / \mathrm{C}++$ code generated using MATLAB's Simulink Coder. The code executed by each robot implements the communication module, state estimation, and the control law while the plant is the physical robot hardware. Fig. 9 shows a diagram of the embedded system operating onboard of each robot.

Physical experiments take place in a $3 \mathrm{~m}$ x $8 \mathrm{~m}$ rectangular section of a carpeted hallway. An additional computer is used to act as the central command to synchronously start each experiment. Fig. 13 shows our physical experiment setup. To obtain accurate position information, obstacles were set up along both lengths of the hallway and were used for localization. In this case, the particle filtering is used for low run-time complexity. Fig. 14 shows the map of the particle filtering algorithm.

\subsection{Constant time delay}

This section demonstrates the stability of the physical CAVN when there is no delay $\epsilon=0$ (recall our assumption for this paper is homogeneous time delays, then the subscripts $i, j$ are not necessary). 


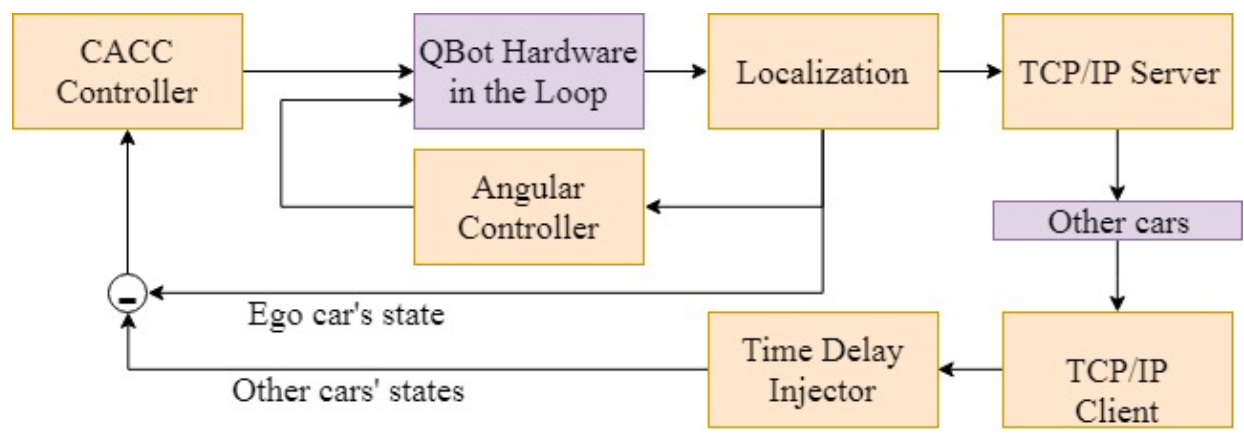

Figure 9: This block diagram shows the software blocks that each robot runs on-board its Raspberry Pi. The physical hardware in the loop is shown in purple.

The used parameters are $h_{\text {eqm }}=1$ with $h_{s t}=0.1 \mathrm{~m}, h_{g o}=2.2 \mathrm{~m}$ and $v_{\max }=0.25 \mathrm{~m} / \mathrm{s}$. The flow equilibrium car gap and velocity are therefore $h_{\text {eqm }}=1 \mathrm{~m}$ and $v_{\text {eqm }}=0.09718 \mathrm{~m} / \mathrm{s}$. The time headway is over 10 seconds. The set-up includes four robots in the initial positions $3 \mathrm{~m}, 2 \mathrm{~m}, 1.3 \mathrm{~m}$, and $-0.3 \mathrm{~m}$. These initial positions capture the cases where the vehicles are: (i) at the equilibrium car gap, (ii) too close to each other, and (iii) too far from one and another, respectively. Fig. 10 shows the stable platoon of four vehicles travelling in flow equilibrium. The control gains are

$$
\alpha=\left[\begin{array}{llll}
0 & 0 & 0 & 0 \\
.8 & 0 & 0 & 0 \\
.8 & .8 & 0 & 0 \\
.8 & .8 & .8 & 0
\end{array}\right] \quad \beta=\left[\begin{array}{llll}
0 & 0 & 0 & 0 \\
.8 & 0 & 0 & 0 \\
.5 & .5 & 0 & 0 \\
.2 & .2 & .2 & 0
\end{array}\right] .
$$

Then the critical time delay value is computed using Theorem 4.1 to verify the stability of the physical CAVN system. The time delay increases to $\epsilon=1$ second. Since the critical time delay value for our control gains was 0.5135 second and is less than the imposed time delay, the dynamic of the experiment in Fig. 11 is unstable. When a different set of control gains

$$
\alpha=\left[\begin{array}{llll}
0 & 0 & 0 & 0 \\
.1 & 0 & 0 & 0 \\
.1 & .1 & 0 & 0 \\
.1 & .1 & .1 & 0
\end{array}\right] \quad \beta=\left[\begin{array}{cccc}
0 & 0 & 0 & 0 \\
.2 & 0 & 0 & 0 \\
.1 & .1 & 0 & 0 \\
.05 & .05 & .05 & 0
\end{array}\right]
$$

are used, the resulting dynamics for the CAVN system (delayed by 1 second) is stable, as shown in Fig. 12. The adjusted control gains give

$$
\epsilon^{*}=\min \{4.4944,3.4608,3.0910\}=3.091>1 .
$$

\subsection{Time-varying delay}

The semi-discretization method predicts plant stability for CAVN systems with time-varying delays. Through the stability region charts in Fig. 3 and 6, important knowledge is obtained for the periodic time-varying delay functions of the type

$$
\epsilon(t)=a-b(1-\cos (\omega t+c))
$$



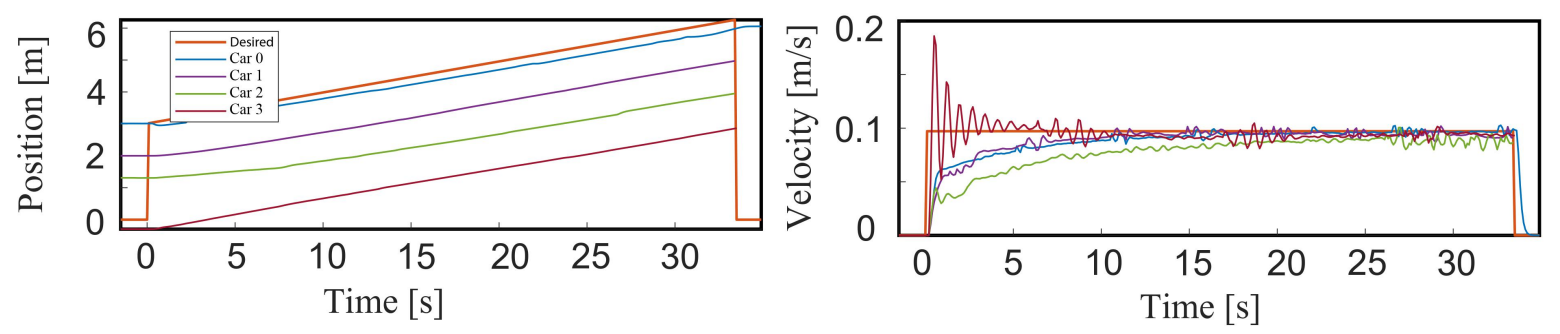

Figure 10: Position and velocity signals of the CAVN system. The system is plant stable when there are no delays. The desired curve shows the perfect leader trajectory at flow equilibrium as a visual reference to the experimental results.
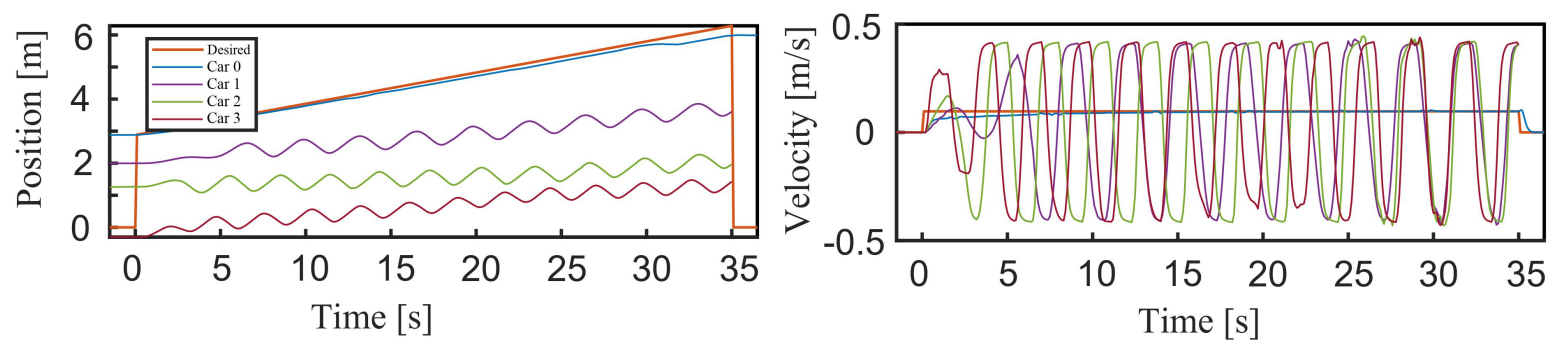

Figure 11: Position and velocity signals show that if $\epsilon=1>0.5135=\epsilon^{*}$, that is, the constant delay is greater than the critical time delay value, then the system is plant unstable; as predicted by Theorem 4.1. The desired curve shows the perfect leader trajectory at flow equilibrium as a visual reference to the experimental results.
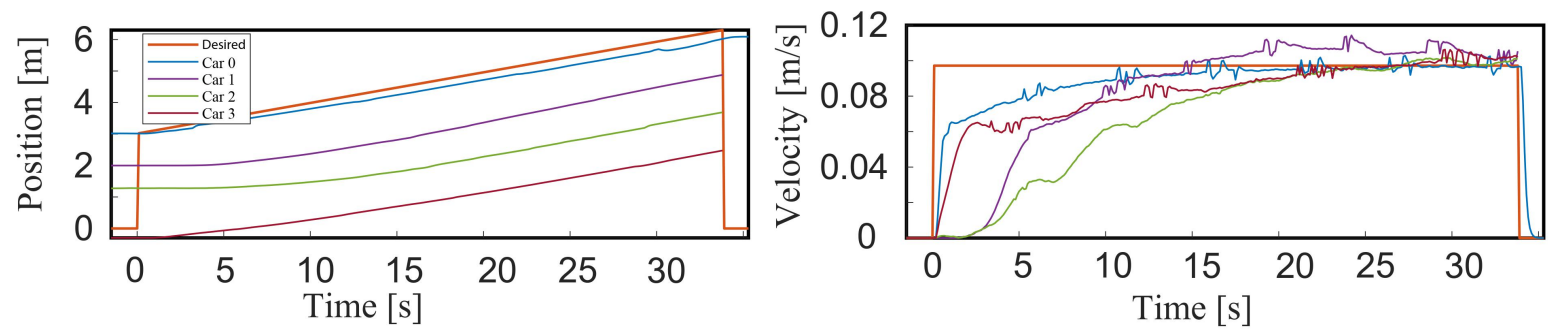

Figure 12: Position and velocity signals shows that if $\epsilon=1<3.091=\epsilon^{\star}$, that is, the constant delay is less than the critical time delay value, then the system is plant stable; as predicted by Theorem 4.1. The desired curve shows the perfect leader trajectory at flow equilibrium as a visual reference to the experimental results.

increases stability margins and engender non-trivial stability boundaries which cannot be fully described using a constant delay approximation. For this subsection, homogeneous control gains $\alpha=0.3$ and $\beta=0.27$ with $a=1$ are used. With $a=1$, the maximum delay is hold at 1 second. Then the time-varying delay dynamics in the $b c$-plane when $\omega=1$ (Fig. 3b) is obtained. In Fig. 15 the amplitude of the time-varying delay function increases from 0.15 to 0.48 and this increase in amplitude gradually stabilizes the CAVN system. This observation is intuitive because as the amplitude increases, the average of the delay function $\epsilon(t)$ decreases. In the extreme case of very slow periodic delay functions, a large amplitude could reduce the time delay to zero for a 


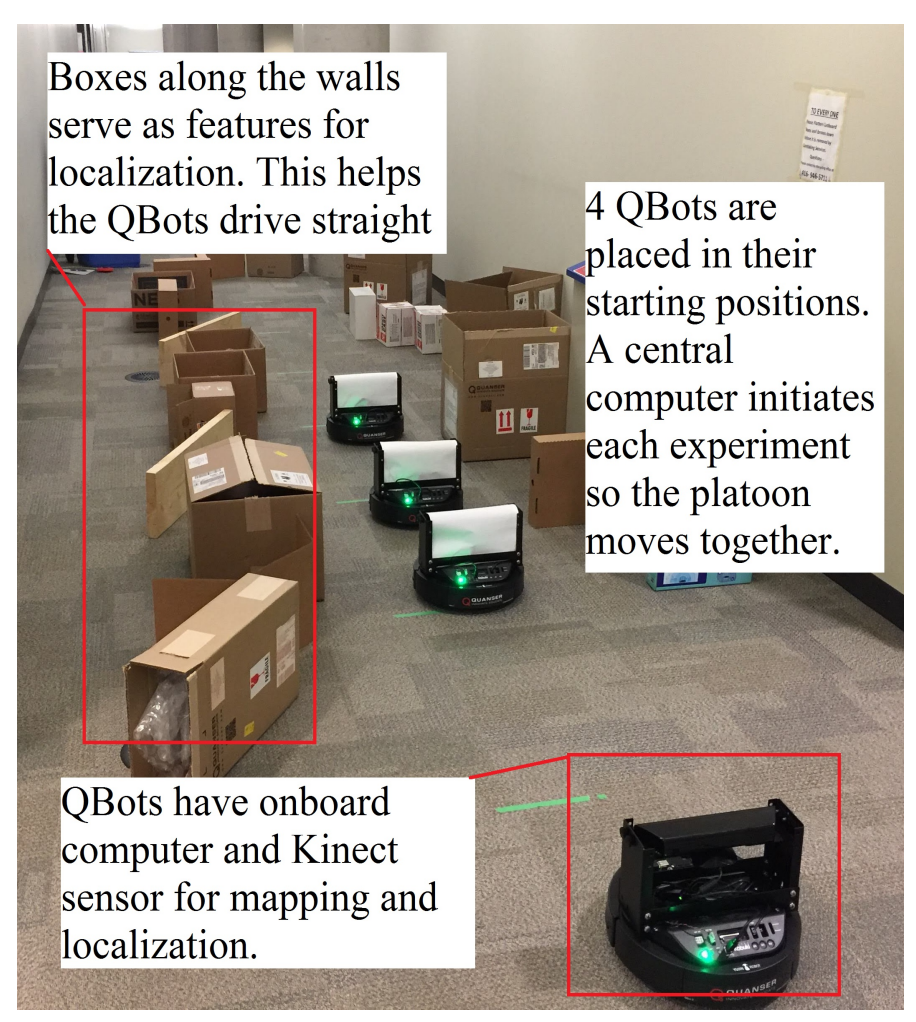

Figure 13: The experimental set up.

sufficiently long period of time such that the CAVN system reaches equilibrium before the time delay can cause instability. This explanation can explain the increase of the stability margin in Fig. 6. The experiment indicates that the experimental threshold for stability is near $b=0.48$. Although the stability boundary obtained via simulation versus experiment was not the same, the effect of increasing amplitude $(b)$ is clear.

Next, the "unstable band" in the bw-plane of Fig. 3a is verified. Ideally, a point on the $b w$-plane inside the "unstable band" is selected the unstable dynamics. However, the previous experiment (Fig. 15) shows that the stability boundary of the physical system was different from the one predicted in Fig. 3b. Then, slightly different parameters should be selected to show that frequency of the time-varying delay function can impact the stability of the CAVN system. In order to rule out the effects that a small value of $b$ keeps the CAVN system unstable, $b=0.48$ is fixed first to get a stable CAVN system. Then the frequency $\omega$ is adjusted via trial and error, and it is found that $\omega=4.2$ indeed destabilizes the CAVN system. The experiments do not confirm the exact shape of the hypothesized "unstable band" in Fig. 3a, the existence of the unstable example $\omega=4.2$ confirms the existence of the "unstable band". Fig. 16 shows that by changing $\omega$ from 1 to 4.2, the adjustment causes the CAVN system to become unstable. This result is particularly important for the multiagent community because of the empirical evidence that bounded timevarying delay functions cannot always be trivially replaced by a constant time delay with a value equal to the maximum delay of the original time-varying function. 


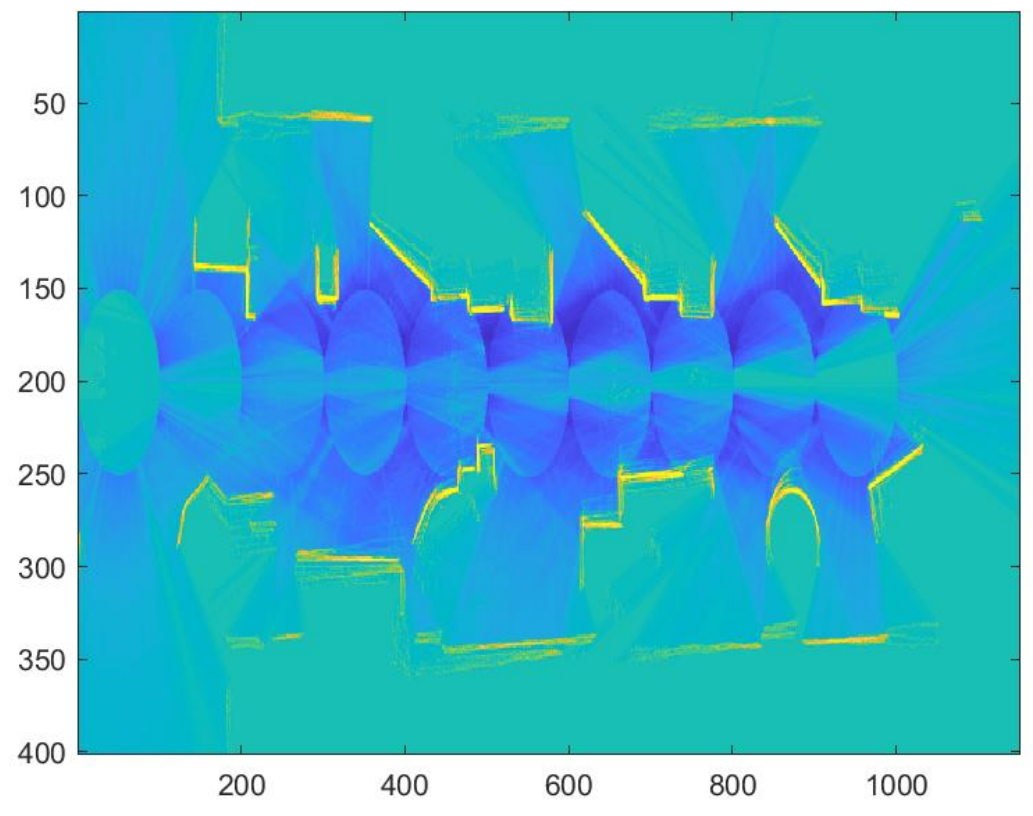

Figure 14: Map used for robot localization. $360^{\circ}$ scans were taken at $1 \mathrm{~m}$ intervals along the length of the experiment area. Dark blue traces represent regions of the map that are highly likely of being unoccupied, and yellow regions represent areas highly likely of being occupied.

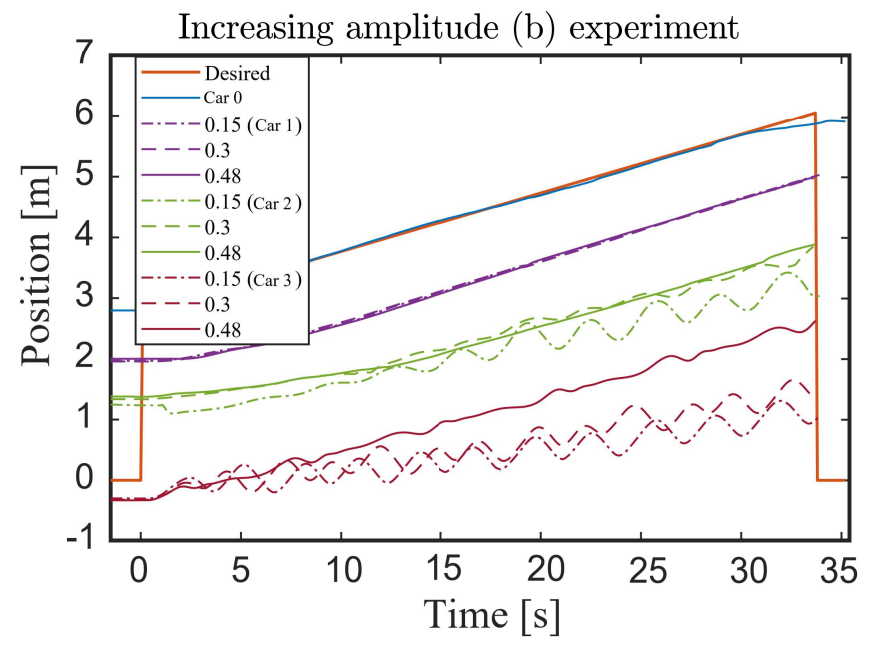

Figure 15: The figure shows that increasing amplitude in the periodic time-varying delay function (b) improves plant stability of a CAVN system. The periodic time-varying delay function is $\epsilon(t)=$ $a-b(1-\cos (\omega t+c))$. The parameters $a=1, \omega=1$ and $c=0$ are used. The values of $b$ such that $2 b<a$ to keep $\epsilon(t)>0 \forall t$. The desired curve shows the perfect leader trajectory at flow equilibrium as a visual reference to the experimental results.

\section{3 $\quad$ String stability}

The experimental demonstration of string stability uses an empirical method described by previous literature as amplification gain estimation [36]. The method produces an approximate plot of the 

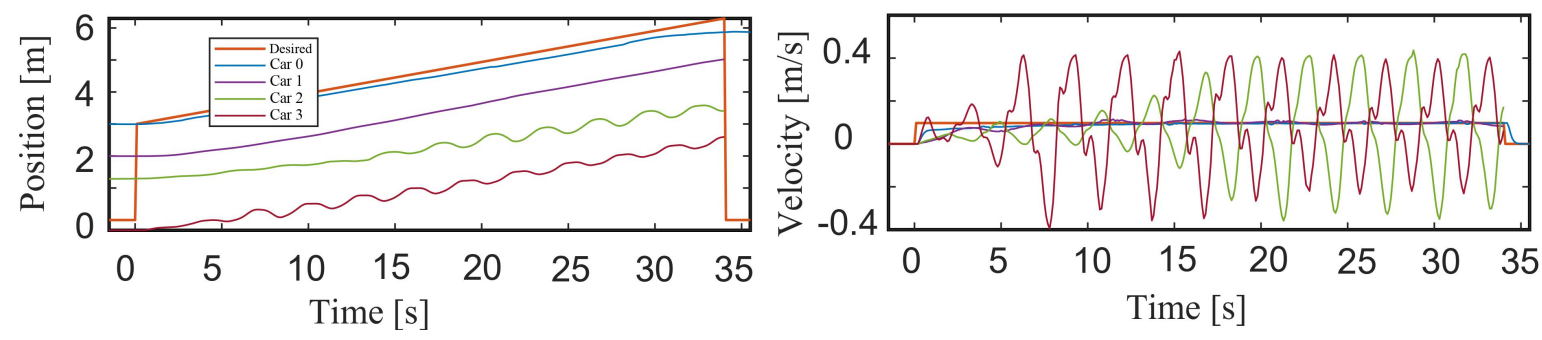

Figure 16: Position and velocity signals show that by adjusting only the frequency of the periodic time-varying delay function, the CAVN system becomes unstable. The parameters $a=1, b=0.48$, and $c=0$ produce stable CAVN dynamics under $\omega=4.2$ in the previous experiment (Fig. 15).

$\left|T_{3,0}(j \omega)\right|$. First the system reaches the flow equilibrium, then pure sinusoidal velocity disturbances are injected into the leader. Specifically, the velocity error $\tilde{v}_{0}=v_{a m p} \sin (\omega t)$. The parameter $v_{a m p}=0.07<v_{\text {eqm }}$ is fixed and $\omega$ vary from 0 to $2 \pi$. (43) is used to infer about the string stability of the system. Fig. 17 shows string stability with constant delays.
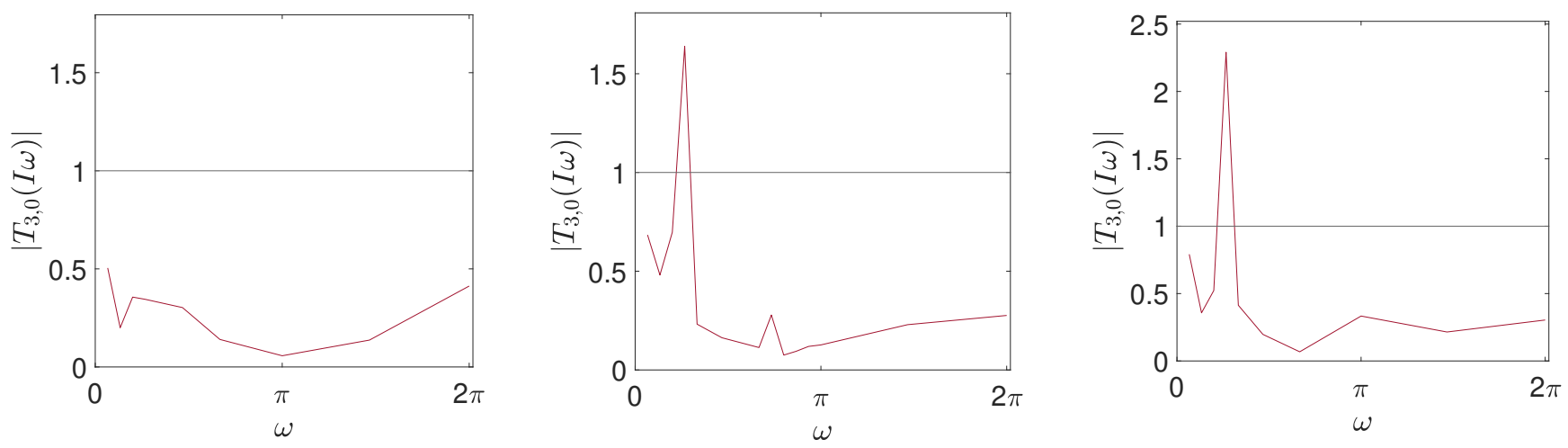

Figure 17: The curve of $\left|T_{3,0}(I \omega)\right|$ with (Left) $\epsilon_{\text {avg }}=1.2$ (Mid.) $\epsilon_{\text {avg }}=1.3$ (Right) $\epsilon_{\text {avg }}=1.67$. System (6) is string stable in (a) because the the maximum value of $\left|T_{3,0}(I \omega)\right|$ is less than 1 for all $\omega>0$ and it is string unstable in (b)-(c) since the supremum of $\left|T_{3,0}(I \omega)\right|$ is bigger than 1 for $\omega \in(0, \infty)$.

\section{Conclusions}

In this paper, a stability framework is proposed to study the CAVN system with constant and timevarying communication delays. In the case of constant time delay, explicit sufficient conditions of the system parameters for the stability of the flow equilibrium are provided. The time-varying delays use harmonic functions to change the velocity profile of the CAVN. Hence, the monodromy operator corresponding to the linear delayed system about the flow equilibrium is an infinitedimensional operator. Consequently, stability conditions cannot be given as closed-form functions of the system parameters explicitly. To address this, the semi-discretization method is used for the stability analysis of the flow equilibrium. The semi-discretization method allows the construction of stability charts for the flow equilibrium over different parameter spaces. Conditions for string 
stability are also theorized and made available to analyze our numerical and experimental CAVN system.

Through numerical and experimental examples, it is observed that: (i) updated control gains can stabilize the flow equilibrium; (ii) for most choices of periodic time-varying delay function, the platoon exhibited a larger stability margin than constant delay; and (iii) very specific choices of periodic time-varying delay functions can cause the platoon to become unstable. Then, experimentation with a connected autonomous robot system was conducted to explore: (i) the influence of the amplitude and frequency of the time-delay function on stabilizing the platoon, increasing amplitude has a stabilizing effect on the platoon while there exists a frequency band for which the platoon is destabilized); and (ii) the string stability of the time-delayed platoon. Future research directions include expanding the classes of time-varying delay functions considered and analyzing the stability of the resulting CAVN networks. It is also interesting to design a control system that stabilizes time-varying delayed systems.

\section{Conflict of interest}

The authors declare that they have no conflict of interest.

\section{Statement}

The data that support the findings of this study are available from the corresponding author, upon reasonable request.

\section{References}

[1] S. Parkinson, P. Ward, K. Wilson, and J. Miller, "Cyber threats facing autonomous and connected vehicles: Future challenges," IEEE Transactions on Intelligent Transportation Systems, vol. 18, pp. 2898-2915, 2017.

[2] L. Xu, L. Y. Wang, G. Yin, and H. Zhang, "Impact of communication erasure channels on the safety of highway vehicle platoons," IEEE Transactions on Intelligent Transportation Systems, vol. 16, pp. 1456-1468, 2014.

[3] R. Sipahi, F. Atay, and S. Niculescu, "Stability of traffic flow behavior with distributed delays modeling the memory effects of the drivers," SIAM Journal on Applied Mathematics, vol. 68, pp. 738-759, 2008.

[4] R. Hult, G. R. Campos, E. Steinmetz, L. Hammarstrand, P. Falcone, and H. Wymeersch, "Coordination of cooperative autonomous vehicles: Toward safer and more efficient road transportation," IEEE Signal Processing Magazine, vol. 33, pp. 74-84, 2016.

[5] S. E. Shladover, "Connected and automated vehicle systems: Introduction and overview," Journal of Intelligent Transportation Systems, vol. 22, pp. 190-200, 2018. 
[6] G. Orosz, "Connected cruise control: modelling, delay effects, and nonlinear behaviour," Vehicle System Dynamics, vol. 54, pp. 1147-1176, 2016.

[7] R. Sipahi, F. Atay, and S. Niculescu, "Stability of traffic flow behavior with distributed delays modeling the memory effects of the drivers," Vehicle System Dynamics, vol. 68, pp. 1147-1176, 2007.

[8] A. Petrillo, A. Salvi, S. Santini, and A. S. Valente, "Adaptive multi-agents synchronization for collaborative driving of autonomous vehicles with multiple communication delays," Transportation Research Part C Emerging Technology, vol. 86, pp. 372-392, 2018.

[9] M. Di Bernardo, A. Salvi, and S. Santini, "Distributed consensus strategy for platooning of vehicles in the presence of time-varying heterogeneous communication delays," IEEE Transactions on Intelligent Transportation Systems, vol. 16, pp. 102-112, 2014.

[10] G. Fiengo, D. G. Lui, A. Petrillo, S. Santini, and M. Tufo, "Distributed robust pid control for leader tracking in uncertain connected ground vehicles with v2v communication delay," IEEE/ASME Transactions on Mechatronics, vol. 24, no. 3, pp. 1153-1165, 2019.

[11] M. di Bernardo, P. Falcone, A. Salvi, and S. Santini, "Design, analysis, and experimental validation of a distributed protocol for platooning in the presence of time-varying heterogeneous delays," IEEE Transactions on Control Systems Technology, vol. 24, no. 2, pp. 413-427, 2016.

[12] L. Xu, W. Zhuang, G. Yin, and C. Bian, "Stable longitudinal control of heterogeneous vehicular platoon with disturbances and information delays," IEEE Access, vol. 6, pp. 69794-69806, 2018.

[13] D. Zhang, Z. Xu, G. Feng, and H. Li, "Asynchronous resilient output consensus of switched heterogeneous linear multivehicle systems with communication delay," IEEE/ASME Transactions on Mechatronics, vol. 24, no. 6, pp. 2627-2640, 2019.

[14] Z. Abdollahi Biron, S. Dey, and P. Pisu, "Real-time detection and estimation of denial of service attack in connected vehicle systems," IEEE Transactions on Intelligent Transportation Systems, vol. 19, no. 12, pp. 3893-3902, 2018.

[15] Y. Feng, C. Yu, and H. X. Liu, "Spatiotemporal intersection control in a connected and automated vehicle environment," Transportation Research Part C: Emerging Technologies, vol. 89, pp. 364-383, 2018.

[16] Y. Bian, Y. Zheng, W. Ren, S. E. Li, J. Wang, and K. Li, "Reducing time headway for platooning of connected vehicles via v2v communication," Transportation Research Part C: Emerging Technologies, vol. 102, pp. 87-105, 2019.

[17] J. Lioris, R. Pedarsani, F. Y. Tascikaraoglu, and P. Varaiya, "Platoons of connected vehicles can double throughput in urban roads," Transportation Research Part C: Emerging Technologies, vol. 77, pp. 292-305, 2017.

[18] W. B. Qin and G. Orosz, "Scalable stability analysis on large connected vehicle systems subject to stochastic communication delays," Transportation Research Part C: Emerging Technologies, vol. 83, pp. 39-60, 2017. 
[19] Y. Zhou and S. Ahn, "Robust local and string stability for a decentralized car following control strategy for connected automated vehicles," Transportation Research Part B: Methodological, vol. 125, pp. 175-196, 2019.

[20] Y. Yang, D. Xu, M. Tan, and X. Dai, "Stochastic stability analysis and control of networked control systems with randomly varying long time-delays," Fifth World Congress on Intelligent Control and Automation (IEEE Cat. No. 04EX788), vol. 2, pp. 1391-1395, 2004.

[21] Y. Li, C. Tang, S. Peeta, and Y. Wang, "Nonlinear consensus-based connected vehicle platoon control incorporating car-following interactions and heterogeneous time delays," IEEE Transactions on Intelligent Transportation Systems, vol. 20, no. 6, pp. 2209-2219, 2019.

[22] H. Xu, J. Sarangapani, and F. Lewis, "Stochastic optimal control of unknown networked control systems in the presence of random delays and packet losses," Automatica, vol. 48, p. 1017-1030, 062012.

[23] A. Petrillo, A. Pescapé, and S. Santini, "A secure adaptive control for cooperative driving of autonomous connected vehicles in the presence of heterogeneous communication delays and cyberattacks," IEEE Transactions on Cybernetics, pp. 1-16, 2020.

[24] I. Al-Darabsah, M. Al Janaideh, and S. A. Campbell, "Stability of connected autonomous vehicle networks with commensurate time delays," in 2021 American Control Conference (ACC), pp. 3308-3313, IEEE, 2021.

[25] T. Insperger and G. Stépán, "Semi-discretization method for delayed systems," Int J Numer Methods Eng, vol. 55, pp. 503-518, 2002.

[26] T. Insperger and G. Stépán, Semi-discretization for time-delay systems: stability and engineering applications. Springer Science \& Business Media, 2011.

[27] B. Balachandran, T. Kalmár-Nagy, and D. E. Gilsinn, Delay differential equations. Springer, 2009 .

[28] F. Hartung, T. Insperger, G. Stépán, and J. Turi, "Approximate stability charts for milling processes using semi-discretization," Appl Math Comput, vol. 174, pp. 51-73, 2006.

[29] I. Győri, F. Hartung, and J. Turi, "Preservation of stability in delay equations under delay perturbations," Journal of Mathematical Analysis and Applications, vol. 220, pp. 290-312, 1998.

[30] R. Szalai and G. Orosz, "Decomposing the dynamics of heterogeneous delayed networks with applications to connected vehicle systems," Phys Rev E, vol. 88, no. 4, p. 040902, 2013.

[31] G. Orosz and S. P. Shah, "A nonlinear modeling framework for autonomous cruise control," Dynamic Systems and Control Conferencef, pp. 467-471, 2012.

[32] A. H. Nayfeh and B. Balachandran, Applied nonlinear dynamics: analytical, computational, and experimental methods. John Wiley \& Sons, 2008. 
[33] D. Swaroop and J. K. Hedrick, "String stability of interconnected systems," IEEE Trans Automat Contr, vol. 41, no. 3, pp. 349-357, 1996.

[34] D. Hajdu, L. Zhang, T. Insperger, and G. Orosz, "Robust stability analysis for connected vehicle systems," IFAC-PapersOnLine, vol. 49, no. 10, pp. 165-170, 2016.

[35] L. Zhang and G. Orosz, "Motif-based design for connected vehicle systems in presence of heterogeneous connectivity structures and time delays," IEEE Transactions on Intelligent Transportation Systems, vol. 17, no. 6, pp. 1638-1651, 2016.

[36] W. B. Qin and G. Orosz, "Experimental validation of string stability for connected vehicles subject to information delay," IEEE Transactions on Control Systems Technology, vol. 28, no. 4, pp. 1203-1217, 2020. 


\section{Supplementary Files}

This is a list of supplementary files associated with this preprint. Click to download.

- NODYresponsesu.pdf 